\title{
EXERCISE PREVENTS AGE-RELATED MEMORY DECLINE: THE ROLE OF NEUROTROPHIC FACTORS
}

\author{
Arina Windri Rivarti ${ }^{1}$, Lilik Herawati ${ }^{2}$, Hanik Badriyah Hidayati ${ }^{3}$ \\ Correspondence: hanikhidayati@fk.unair.ac.id \\ ${ }^{I}$ Master Program of Basic Medical Science, Faculty of Medicine, Universitas Airlangga, Surabaya, Indonesia \\ ${ }^{2}$ Departemen of Physiology, Faculty of Medicine, Universitas Airlangga, Surabaya, Indonesia \\ ${ }^{3}$ Departement of Neurology, Faculty of Medicine, Universitas Airlangga, Surabaya, Indonesia
}

Article History:

Received: November 12, 2019

Accepted: June 25, 2020

Published: July 1, 2020

\section{Cite this as:}

Rivarti AW, Herawati L, Hidayati $H B$. Exercise prevents age-

related memory decline: the role of neurotrophic factors. Malang Neurology Journal; 2020.6:8894.

http://dx.doi.org/10.21776/ub.mnj .2020.006.02.8

\section{ABSTRACT}

Increasing aging population causes an increased prevalence of neurodegenerative diseases such as dementia that is associated with memory decline. Developing strategies for the prevention and therapy of age-related dementia is important to reduce the burden of treatment costs. Physical exercise is known to prevent cognitive decline and improve cognitive abilities. Physical exercise with moderate intensity for at least 150 minutes/week or 30 minutes/day for 5 days can reduce the incidence of degenerative diseases in the elderly. Thus, physical exercise appears as a simple, inexpensive, and affordable non-pharmacological therapy for most people. The processes of neurogenesis and neuronal survival involve the role of neurotrophic factors including BDNF, IGF-1 and VEGF, which are the three main neurotrophic factors that are known to increase after exercise. Many publications discuss about these neurotrophic factors, but their mechanism of signals and changes related to aging and exercise have not been completely studied. The purpose of this review is to discuss the mechanism of signals and changes of neurotrophic factors (focuses on BDNF, IGF-1, and VEGF) related to aging and exercise.

Keywords: Exercise, aging, BDNF, IGF-1, VEGF

\section{Introduction}

Aging is unavoidable and unalterable process. The increasing aging population in the world is caused by improved medical care and decreased fertility. World Health Organization reported that there were 900.9 million people aged 65 years or above in 2015 and the number was expected to increase up to 2092 million by $2050 .{ }^{1}$ Aging is the major risk factor for neurodegenerative diseases, such as dementia which is initially characterized by memory decline. $^{2}$ The population of dementia will continue to increase along with the increasing number of aging population. There are approximately 36.6 million people suffering from dementia in 2010, 50 million people in 2020 , and it was expected to double up by $2030 .{ }^{3,4}$ Therefore, one of the greatest health threats in many countries is agingrelated dementia, which costed US $\$ 818$ billion in 2015 worldwide. ${ }^{4}$

Developing strategies for the prevention and treatment of age-related dementia is important to reduce the burden of treatment costs. Unfortunately, until now there has been no effective pharmacological therapy for aging- related dementia. Therefore, the development of nonpharmacological prevention and treatment is a potential alternative therapy for these neurodegenerative disorders. Physical exercise can be an inexpensive and affordable non-pharmacological therapy in preventing age-related cognitive decline. Moderate intensity physical exercise for at least 150 minutes/week or 30 minutes/day for 5 days can reduce the incidence of degenerative diseases in the elderly. ${ }^{5}$ Prior studies have revealed that exercise or physical activity were able to give neurological advantage and preservative effects on the brain, prevent cognitive decline and improve cognitive performance. ${ }^{6-8}$ Research using animal models showed that exercise increased neurotrophic factors, glial cells activity and cerebral blood flow which led to increased neurogenesis and neuron cell survival. ${ }^{6,8-12}$

Neurotrophic factors such as brain derived neurotrophic factor (BDNF), insulin-like growth factor (IGF-1) and vascular endothelial growth factor (VEGF) are known to be the main mediators of neurogenesis in the adult brain. Physical exercise causes an increased BDNF gene expression in the hippocampus. In addition, physical exercise also causes increased levels of IGF-1 and VEGF in the peripheral, subsequently enters the brain through the blood brain barrier and triggers the process of neurogenesis, thus maintaining cognitive function. ${ }^{13}$ These neurotrophic factors including BDNF, IGF-1 and VEGF have been widely studied, with evidence of the large number of publications discussing these neurotrophic factors.However, the mechanism of signals and changes of these neurotrophic factors related to aging and exercise have not been completely studied. In this article the mechanism of signals and changes of these neurotrophic factors related to aging and exercise are highlighted. 


\section{Neurotrophic Factors, Aging Brain, and Exercise}

Neurotrophic factors are proteins that function as mediators for the neuronal proliferation, differentiation, and survival. The three main neurotrophic factors that are known to increase after exercise and can trigger the neuronal growth and proliferation are brain-derived neurotrophic factor (BDNF), insulin-like growth factor-I (IGF-1) and vascular endothelial growth factor (VEGF). ${ }^{14}$ The aging process is associated with a decreased activity of these neurotrophic factors activity.

\section{BDNF, aging brain, and exercise}

Brain-derived neurotrophic factor (BDNF)is a member of neurotrophin family that is expressed in the cortex, hypothalamus, septum, and in adrenergic brain stem nuclei and is highly concentrated in the hippocampus. ${ }^{14,15}$ The high expression of BDNF in the hippocampus is important considering the main function of the hippocampus for learning and memory. ${ }^{16} \mathrm{BDNF}$ play a role for neuronal growth, differentiation, and survival, synaptic plasticity, proliferation of dendritic arbor, axonal sprouting, regulates LTD (long-term depression) and LTP (long-term potentiation). ${ }^{14,17}$ The expression of this neurotrophicfactor in the peripheral and central nervous systems is influenced by stress, nutrition, behavior, and metabolism. ${ }^{17}$

BDNF is derived from pro-isoform BDNF which will form a mature BDNF (mBDNF) protein after undergoing proteolytic breakdown by tissue type plasminogen activator (tPA) in neurons or after being released.BDNF protein will bind to protein-kinase neurotrophin receptors tropomyosine-related kinase (Trk) receptors. The interaction between BDNF and its receptors causes the activation of several major signaling pathwaysthat play a role in stimulating synaptic plasticity, neuronal proliferation, differentiation and survival, includingPI-3 kinase, MAP kinase and phospholipase-c- $\gamma .{ }^{18,19}$ The binding of BDNF with TrkB receptorsubsequently activates cAMPresponsive element-binding protein (CREB). CREB is a transcription factor that plays a role in regulating the process of neuronal proliferation, differentiation and survival. Therefore, CREB plays an important role in neuronal plasticity, memory and learning. ${ }^{18,20,21}$ Contrastingly, pro-BDNF binding with its receptor, p75 neurotrophin receptor (p75 NTR) activates the apoptotic pathway of neurons and glial cells. ${ }^{17}$

Petzold and his colleagues found BDNF protein levels in the hippocampus (without distinguishing between mBDNF and pro-BDNF) were relatively stable in mice aged 3, 5, 7 and 9 months. ${ }^{22}$ This finding was explained by Calabrese and colleagues comparing $\mathrm{mBDNF}$ and pro-BDNF levels in mice aged 18 months and 3 months. Based on the study, there were reduced mature BDNF levels and elevated proBDNF levels in the hippocampus and prefrontal cortex of old mice. ${ }^{23}$ Increased pro-BDNF levels is related to a decreased activity of pro-BDNF cleaving extracellular protease plasmin due to decreased proteolytic activity of tissue type plasminogen activator (tPA). ${ }^{24}$ Therefore, the total BDNF protein in the brain is relatively stable. However, aging will change the ratio ofpro-BDNF and $\mathrm{mBDNF}$ which representsthe decrease of mBDNF levels and increase of pro-BDNF levels. This resulted in an increase of apoptotic pathways for neurons and glial cells and a decrease of neuronal proliferation, differentiation and survival.

In addition to its influence to the BDNF protein, aging also affects the TrkB receptor. Florence and colleagues observed the TrkB expression in pituitary rats at age of 1 month, 2 months, 4 months, 10 months, and 22 months showed a decrease of TrkB expression along with the increase of rat age. ${ }^{25}$ This was also supported by other studies that presented a decreased expression of TrkB in the hippocampus along with the increased age of mice. ${ }^{22}$

Exercise can cause an increase of BDNF levels. Marlatt and colleagues revealed an increase of neurogenesis and BDNF levels in female mice ( 9 months) after wheel running. The increase of BDNF levels and the number of new neuron cells affected an improvement in memory retention and spatial memory. Although specific molecular mechanisms underlying the increased BDNF gene expression due to exercise were not clearly elucidated, recent studies showed that there was a role of ketone body $D$ - $\beta$-hydroxybutyrate (DBHD) in increasing BDNF gene expression due to exercise. DBHB is a major metabolite which is increasingly secreted in the liver after prolonged exercise. DBHB is produced from acetyl coA derived from the $\beta$-oxidation of fatty acids in the liver. DBHD from the liver enters the blood circulation and enter the brain to be used as an energy source. In addition, DBHD is known to inhibit histone deacetylation (HDAC), specifically HDAC2 and HDAC3 that causes an increased BDNF gene expression. ${ }^{26}$ Therefore, exercise leads to the rise of the expression of BDNF genes through HDAC inhibition by DBHD.

Exercise is also known to be able to inhibit the activity of enzymes $\beta$ and $\gamma$ secretases in neurons through BDNF. These enzymes function to break down amyloid precursor protein (APP) to produce amyloid $\beta$ peptide $(A \beta)$, which is a toxic substance that causes age-related neurodegenerative diseases, such as Alzheimer's disease. Exercise and BDNF cause an increase in the activity of $\alpha$ secretase. The $\alpha$ secretase is one of the three types of secretase which roles in breaking down amyloid precursor proteins and forming sAPP $\alpha$. sAPP $\alpha$ is a secreted form of APP which will be secreted outside neuron cells.sAPP $\alpha$ is also known as intracellular neuroprotective APP peptide. In addition, $\beta$ secretase activity will be inhibited by $\operatorname{sAPP} \alpha$, consenquently preventing the formation and accumulation of $\mathrm{A} \beta$. Therefore, exercise and BDNF have neuroprotective effects by increasing $\alpha$ secretase activity. ${ }^{27}$

IGF-1, aging brain, and exercise

Insulin-like growth factor-1 (IGF-1) is a major growth factor and its production is stimulated by growth hormone $(\mathrm{GH})$. IGF-1 is mainly produced in the liver and is subsequently bound to IGF binding protein (IGFBP) in the blood circulation before eventually entering the blood brain barrier into the central nervous system.26-28 The ability of IGF-1 penetration to the blood-brain barrier explains the relationship between IGF-1 levels in circulation and IGF-1 levels in cerebrospinal fluid. Decreased IGF-1 levels in the circulation will cause a decrease of IGF-1 levels in the cerebrospinal fluid.27 IGF-1 can cross the blood-brain barrier through 3 mechanisms: (1) by binding to IGF-1 
receptors in endothelial cells and then taken directly by neuron cells or through astrocyte cells and afterward transferred to neuron cells; (2) by binding to low-density lipoprotein receptor-related protein-2 (LRP2) into the cerebrospinal fluid (CSF); and (3) by metalloproteinase 9 activity which will break IGF-1 and IGFBP bonds to form

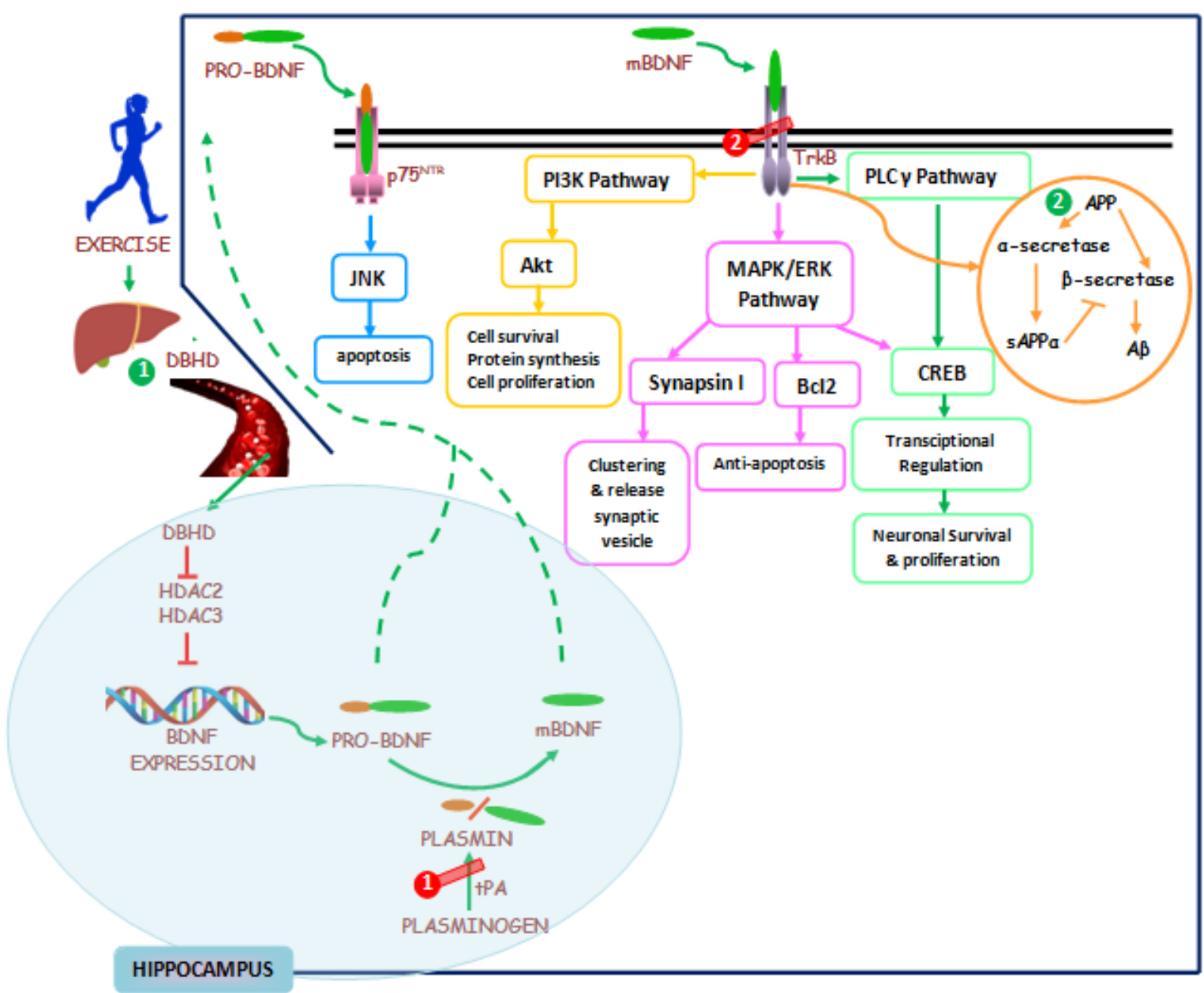

Figure 1. Aging (number with a red circle) causes: (1) an increase of pro-BDNF levels compared to BDNF due to decreased activity of tPA, which results in increased cell apoptosis and (2) decreased expression of TrkB receptors and decreased signaling pathways that function as neuronal survival, proliferation and antiapoptotic pathway. Exercise (number with a green circle) is able to counteract the effects of aging by: (1) increasing DBHD secretion by the liver which will cause increased BDNF expression, thereby increasing BDNF signaling activity and (2) increasing $\alpha$ secretase activity by inhibiting the activity of $\beta$ secretase to form $A \beta$.

free IGF-1 that can enter the central nervous system (CNS). 26,29-31 IGF-1 which has crossed the blood-brain barrier plays a role in increasing BDNF activity, activating c-Fos and activating various major signaling pathways similar toBDNF. ${ }^{14,28}$ Thus, IGF-1 activity in the brain is important for neuronal proliferation, differentiation and survival, neurotransmission, synaptic density, synaptic plasticity, and adult neurogenesis.

Aging causes a decrease of IGF-1 and IGF-1R levels. ${ }^{29,30}$ Study by Hyun et al. disclosed a decrease of IGF-1 and IGF-1 receptor levels in the hippocampus and somatosensory cortex of mouse at age of 24 months compared to mouse at age of 6 months. ${ }^{31}$ Other studies presented a decrease of IGF-1 levels and IGF-1R expression in gerbil olfactory bulbs at age of 24 months compared to age of 3 and 6 months. ${ }^{32}$ Thus, a decrease of IGF-1 levels and IGF-1R expression causes a decreased activity of BDNF activity and the activity of various major signaling pathways associated with neuronal survival, neurogenesis and synaptic plasticity. This results in decreased cognitive function and memory in the elderly.

Age-related decrease of IGF-1also causes a decrease of expression and NMDA receptor activity. NMDA receptors are receptors that play a role in maintaining long term potentiation (LTP) expression and generating action potentials in neurons which play an important role in hippocampal-dependent learning and memory. The NMDA receptor is a tetramer that consists of different subunits. Within the hippocampus, the NMDA receptor consists of two GluN1 subunits and two other subunits consisting of GluN2A or GluN2B. Aging causes a significant decrease of GluN2B expression. This subunit reduction is associated with age-related cognitive disorders. This is proved by overexpression of GluN2B correcting cognitive impairment in old rats. IGF-1 supplementation in older rats successfully corrected the expression of GluN2A/GluN2B, suggesting that IGF-1 played an important role in NMDA expression and activity. ${ }^{33}$ 
In addition to its effect to NMDA expression, decreased IGF-1 levels and age-related cellular sensitivity to IGF-1 also play a role in the accumulation of dangerous compounds such as $\beta$-amyloid peptides $(\beta A)$ in the brain. $\beta \mathrm{A}$ is a compound that plays a role in age-related dementia, including alzheimer's and vascular dementia. IGF-1 plays a role in protecting nerve cells from the toxicity of $\beta \mathrm{A}$, increasing the release of these compounds from cells and

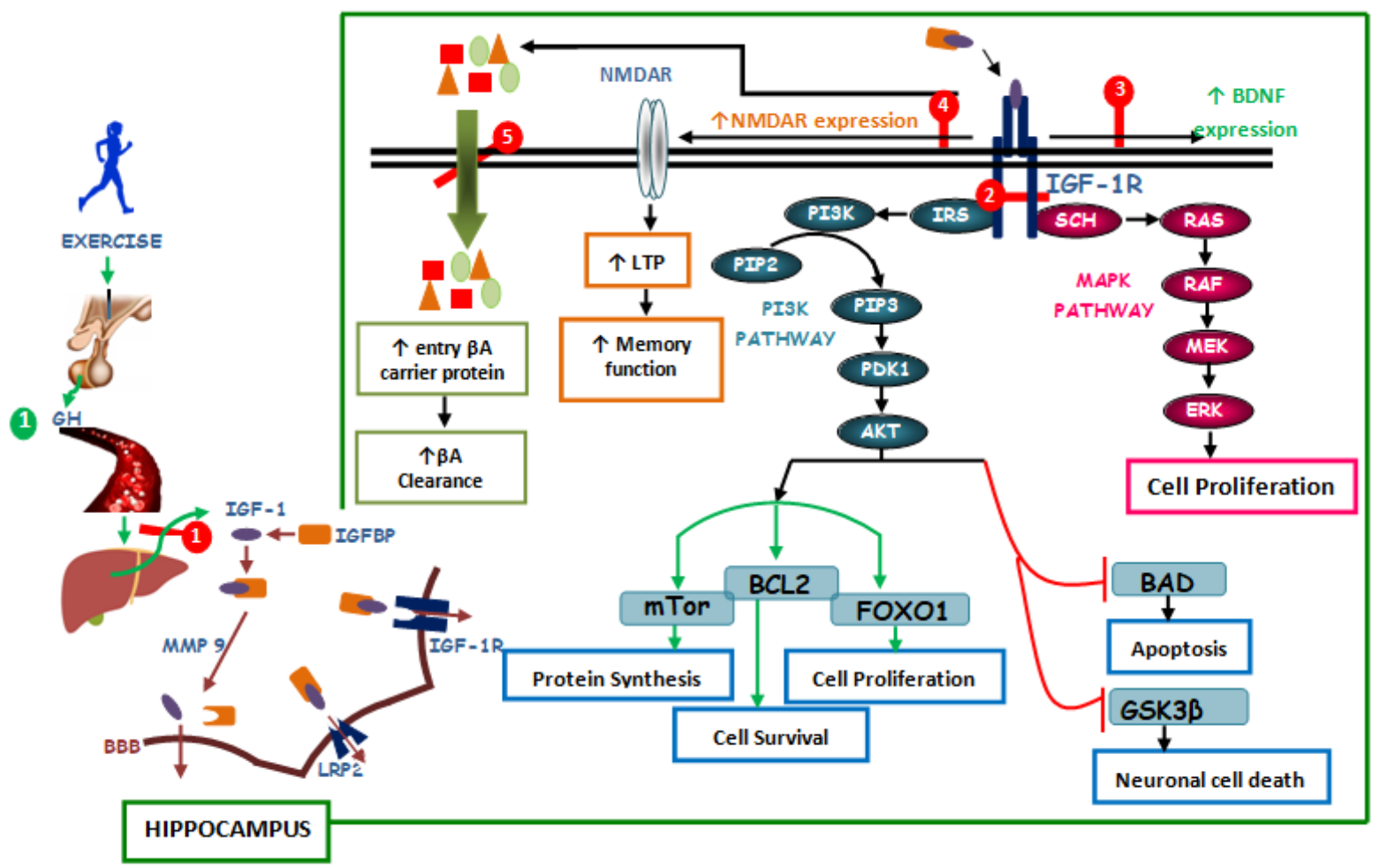

Figure 2. Aging (number with a red circle) causes: (1) decreased IGF-1 levels and (2) decreased IGF-1R expression, thus inhibits proliferation, cell survival, and antiapoptosis signaling pathway; (3) inhibits increased BDNF expression; (4) inhibits increased NMDAR expression; and (5) inhibits clearance from $\beta$ A. Exercise (number with a green circle) can counteract the effects of aging through: (1) an increase of growth hormone which causes an increase in IGF-1 levels by the liver.

stimulating the clearance of these compound in brain tissues. Another newly revealed mechanism is IGF-1 increases the entry of $\beta$ A carrier proteins, such as albumin, transthyretin and apolipoprotein $\mathrm{J}$ into cells, and subsequently binds to $\beta \mathrm{A}$ and carries $\beta \mathrm{A}$ out of the brain parenchyma into the blood circulation through cerebrospinal fluid. ${ }^{34}$

The specific molecular mechanism underlying the improvement of memory by IGF-1 is still unknown, but it is known that IGF-1 corrects age-memory disorders through synapse formation in the hippocampus. Studies on the profile of total synaptic, postsynaptic density (PSD) and multiple spine bouton (MSB) complexes in the CA1 region in the hippocampus of rats aged 4, 18 and 29 months showed that aging caused a decrease of total synapses but retained the length of PSD, and the MSB complex. IGF-1 infusion for 28 days did not improve the reduction in total synapses due to aging. However, it successfully increased the number of MSD synapses and PSD length. ${ }^{35}$ This suggested that both aging and IGF-1 showed complementary functions and affected different parts of the synapse.

Exercise causes an increase of IGF-1 levels by increasing growth hormone $(\mathrm{GH})$ levels. Plasma GH levels will increase within 10-20 minutes after exercise, and $\mathrm{GH}$ is the main regulator of IGF-1 synthesis in the liver. ${ }^{36}$ Many studies have shown that exercise plays a role in increasing
IGF-1 activity. Carro and colleagues showed that wistar rats treated with treadmill running of $17 \mathrm{~m} / \mathrm{min}$ for 1 hour could induce uptake of IGF-1 from blood to neurons in the brain, and accumulation of IGF-1 in neuron cells caused an increase in sensitivity to prolonged afferent stimulus. Systemic injection of IGF-1 in sedentary ratsresembled the effect of exercise on the brain, which showed the same pattern of IGF-1 accumulation in the brain after exercise or after intracarotid injection of IGF-1. The accumulation of IGF-1 in the brain also causes an increase of c-Fos neuronal activity and the number of new neurons in the hippocampus. The mechanism generated by IGF-1 is similar to stimulation of BDNF in the hippocampus. Increased expression of c-Fos was inhibited after exercise, if IGF-1 uptake by brain cells was inhibited by antiserum IGF- $1 .{ }^{37,38,39}$ This suggested that IGF-1 in circulation was important and exercise could initiate an increase of IGF-1 activity in the brain by stimulating IGF-1 uptake in circulation to the brain. ${ }^{37,38}$

\section{VEGF, aging brain, and exercise}

Vascular endothelial growth factor (VEGF) is a hypoxiainduced protein which plays an important role to stimulate the growth and formation of blood vessels. VEGF can be secreted by a variety of cells and tissues, including glial cells, macrophages, endothelial cells, and smooth and skeletal muscle. ${ }^{14}$ VEGF binding with VEGF receptor 2 
(VEGFR2) causes an increase in angiogenic, mitogenic and neurogenic activity. ${ }^{40}$

Studies evaluating the VEGF response in older mice showed that injection of adeno-associated viral vector expressing VEGF (AAV-VEGF) caused a three times increase of vascular density in brain of mice aged 3 and 12 months compared to mice aged 24 months. In addition, there was an increase in the number of endothelial cells and neuroprogenitors in the subventricular area in mice aged 3 and 12 months compared to 24 months. AAV-VEGF injection also increased the expression of VEGF receptor 2 in the brains of mice aged 3 and 12 months, but not in mice

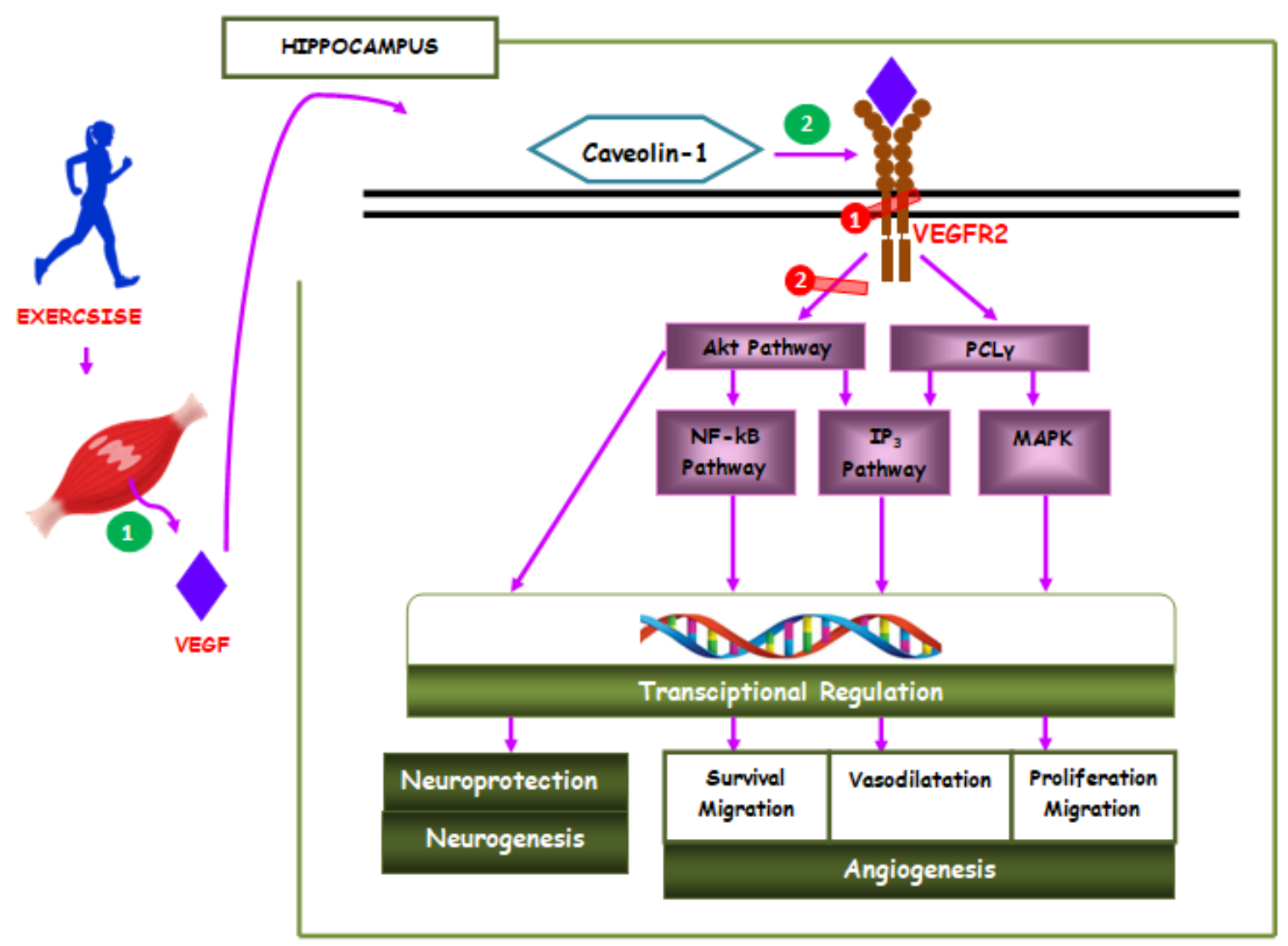

Figure 3. Aging (number with a red circle) causes: (1) decreased VEGFR2 expression and (2) decreased Akt phosphorylation activity induced by VEGF, causing a decrease in signaling pathways of neuroprotective and neurogenesis. Exercise (number with a green circle) is able to counteract the effects of aging by following mechanisms: (1) increasing of VEGF levels by skeletal muscle and (2) increasing caveolin-1 activity, mediating an increase of VEGF expression thereby increasing the activity of the signaling pathway by VEGF.

aged 24 months. ${ }^{40}$ Other studies have proved that there was a decrease in the VEGFR2 protein in the coronary arteries and a decrease in the phosphorylation of Akt (wich involve in PI3-kinase signaling) induced by VEGF in older mice compared with young mice. ${ }^{41}$ This suggested that aging caused a decrease in response to VEGF stimulation and impairment of VEGFR2/PI3-kinase signaling, which was likely due to decreased VEGF receptor function and expression.

Exercise causes an increase of several growth factors including VEGF. There was an increase of neuronal proliferation in the subgranular zone (SGZ) in the hippocampus of mice after having exercise on a running wheel for 1 week. Blocking peripheral VEGF with soluble Flt-1 fusion protein (sFlt) caused a decrease in neuronal proliferation in the hippocampus. This illustrated that peripheral VEGF played an important role in the process of neurogenesis in the hippocampus. ${ }^{42}$ It was estimated that $60-90 \%$ of peripheral VEGF was produced by muscles. Therefore, skeletal muscle may be the main source of VEGF that was involved in exercise-induced neurogenesis. This was proven by research that observed the number of neuronal precursor cells in the hippocampal dentate gyrus of mice with normal skeletal myofiber VEGF production (VEGF ${ }^{\mathrm{f} / \mathrm{f}}$ mice) and transgenic mice with decreased levels

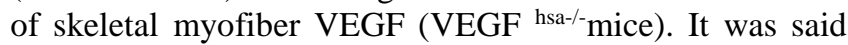
that wheel running for 2 weeks caused increased neurogenesis in VEGF ${ }^{\mathrm{f} / \mathrm{f}}$ mice but no effect was found in VEGF $^{\text {hsa }-/}$ mice. ${ }^{43}$

In addition to increasing VEGFR and neural stem cell (NSCs) activity, treadmill exercise in rats also showed significantly increased Caveolin-1. Caveolin-1 is a protein that mediates cell and tissue regeneration. Inhibition of caveolin-1 by daidzein was reported to decrease caveolin-1 and VEGF levels. It was known that caveolin-1 was involved in following mechanism: (1) caveolin-1 was thought to mediate the VEGF signaling pathway; (2) caveolin-1/VEGF signaling pathways via VEGFR2 after exercise induced NSC differentiation and proliferation, (3) decreased caveolin-1 activity caused a decrease in VEGF expression, thus inhibiting stimulation of VEGFR2 phosphorylation by VEGF and inhibits several major signaling pathways such as PLC $\gamma 1$, AKT, and ERK. ${ }^{44,45}$ 


\section{Conclusion}

Age-related neurodegenerative disease can becaused by a decreased activity, levels, and expression of neurotrophic factors such as BDNF, IGF-1, and VEGF. These three factors have a role in increasing neuronal survival and neurogenesis through the activation of various signaling pathways. Generally, exercise stimulates the expression and activity of these neurotrophic factors. Therefore, exercise can be considered as a preventive therapy to prevent agerelated cognitive and memory decline. However, the variation of intensity, the duration, and type of exercise on neuronal survival and neurogenesis needs further discussion. Hence, the research design may develop to gain a better dose of ecxercise that affect neuronal survival and neurogenesis.

\section{Acknowledgement}

The authors thank Study Program ofBasic Medical Science, Department of Physiology, and Department of Neurology, Faculty of Medicine, Universitas Airlangga, for their support and facilities on this work.

\section{References}

1. United Nations, Department of Economic and Social Affairs PD.World population ageing. New York: United Nations; 2015. Available from:https://www.un.org/en/development/desa/populati on/publications/pdf/ageing/WPA2015_Report.pdf

2. Wahl D, Solon-Biet SM, Cogger VC, et al. Aging, lifestyle and dementia. Neurobiol Dis; 2019.130(9):104481. DOI:10.1016/j.nbd.2019.104481

3. Lastri DN, Alwahdy AS. Clinical and radiologic approach to probable mixed dementia (vascular dementia and progressive supranuclear palsy). Malang $\begin{array}{lll}\text { Neurol J; 2020.6(1):46-50. } & \text { J }\end{array}$ DOI:10.21776/ub.mnj.2020.006.01.10

4. Wimo A, Guerchet M, Ali GC, et al. The worldwide costs of dementia 2015 and comparisons with 2010. Alzheimer's Dement; 2017.13(1):1-7. DOI:10.1016/j.jalz.2016.07.150

5. Nelson ME, Rejeski WJ, Blair SN, et al. Physical activity and public health in older adults: recommendation from the american college of sports medicine and the american heart association. Med Sci Sports 2007.39(8):1435-1445. DOI:10.1249/mss.0b013e3180616aa2

6. Vilela TC, Muller AP, Damiani AP, et al. Strength and aerobic exercises improve spatial memory in aging rats through stimulating distinct neuroplasticity mechanisms.Mol Neurobiol; 2017.54(10):7928-7937. DOI:10.1007/s12035-016-0272-x

7. Tsai SF, Ku NW, Wang TF, et al. Long-term moderate exercise rescues age-related decline in hippocampal neuronal complexity and memory. Gerontology; 2018.64(6):551-561. DOI:10.1159/000488589

8. Vanzella C, Neves JD, Vizuete AF, et al. Treadmill running prevents age-related memory deficit and alters neurotrophic factors and oxidative damage in the hippocampus of wistar rats. Behav Brain Res; 2017.334(July):78-85. DOI: 10.1016/j.bbr.2017.07.034
9. Wang S, Chen L, Zhang L, et al. Effects of long-term exercise on Spatial learning, memory ability, and cortical capillaries in aged rats. Med Sci Monit; 2015.21:945-954. DOI:10.12659/MSM.893935

10. Ohtomo R, Kinoshita K, Ohtomo G, et al. Treadmill exercise suppresses cognitive decline and increases white matter oligodendrocyte precursor cells in a mouse model of prolonged cerebral hypoperfusion.Transl Stroke Res; 2019. DOI:10.1007/s12975-019-00734-7

11. Littlefield AM, Setti SE, Priester C, Kohman RA. Voluntary exercise attenuates LPS-induced reductions in neurogenesis and increases microglia expression of a proneurogenic phenotype in aged mice. $J$ Neuroinflammation; DOI:10.1186/s12974-015-0362-0

12. Tsai SF, Chen PC, Calkins MJ, Wu SY, Kuo YM. Exercise counteracts aging-related memory impairment: a potential role for the astrocytic metabolic shuttle. Front Aging Neurosci; 2016.8(3):1-12. DOI:10.3389/fnagi.2016.00057

13. Yau SY, Gil-Mohapel J, Christie BR, So KF. Physical exercise-induced adult neurogenesis: A good strategy to prevent cognitive decline in neurodegenerative diseases? Biomed Res Int. 2014. DOI:10.1155/2014/403120

14. Maass A, Düzel S, Brigadski T, Goerke M, Becke A, Sobieray U. NeuroImage relationships of peripheral IGF-1 , VEGF and BDNF levels to exercise-related changes in memory, hippocampal perfusion and volumes in older adults.Neuroimage; 2016.131:142 154. DOI:10.1016/j.neuroimage.2015.10.084

15. Tapia-arancibia L, Rage F, Givalois L, Arancibia S. Physiology of BDNF : focus on hypothalamic function. Front Neuroendocrinol; 2004.25:77-107. DOI:10.1016/j.yfrne.2004.04.001

16. Prabawati R, Ratnawati R, Rahayu M, Prakosa A. Effect anthocyanin of purple potato gunung kawi on mda levels , expression of caspase-3, and spatial memory function on diabetic. Malang Neurol $\mathbf{J}$; 2019.5(1):34-41. DOI:10.21776/ub.mnj.2019.005.01.6

17. Budni J, Bellettini-Santos T, Mina F, Garcez ML, Zugno AI. The involvement of BDNF, NGF and GDNF in aging and alzheimer's disease. Aging Dis; 2015.6(5):331-341. DOI:10.14336/AD.2015.0825

18. Chen MJ, Russo-neustadt AA. Exercise activates the phosphatidylinositol 3-kinase pathway. Mol Brain Res; 2005.135:181-193.

DOI:10.1016/j.molbrainres.2004.12.001

19. Czuba E, Was M, Steliga A, Morys J. BDNF : A key factor with multipotent impact on brain signaling and synaptic plasticity. Cell Mol Neurobiol; 2018.38:579593. DOI:10.1007/s10571-017-0510-4

20. Zhang J, Cai CY, Wu HY, Zhu LJ, Luo CX, Zhu DY. CREB-mediated synaptogenesis and neurogenesis is crucial for the role of 5-HT1a receptors in modulating anxiety behaviors. Sci Rep; 2016.6(3). DOI:10.1038/srep29551

21. Ortega-Martínez S. A new perspective on the role of the CREB family of transcription factors in memory consolidation via adult hippocampal neurogenesis. Front Mol Neurosci; 2015;8(8):1-12. 
DOI:10.3389/fnmol.2015.00046

22. Petzold A, Psotta L, Brigadski T, Endres T, Lessmann V. Neurobiology of Learning and memory chronic BDNF deficiency leads to an age-dependent impairment in spatial learning. Neurobiol Learn Mem; 2015.120:52-60. DOI:10.1016/j.nlm.2015.02.009

23. Calabrese F, Guidotti G, Racagni G, Riva MA. Neurobiology of aging reduced neuroplasticity in aged rats: a role for the neurotrophin brain-derived neurotrophic factor.Neurobiol Aging; 2013.1-9. DOI:10.1016/j.neurobiolaging.2013.06.014

24. Obiang P, Maubert E, Bardou I, et al. Neurobiology of learning and memory enriched housing reverses ageassociated impairment of cognitive functions and tpadependent maturation of BDNF. Neurobiol Learn Mem; 2011.96(2):121-129. DOI:10.1016/j.nlm.2011.03.004

25. Rage F, Binam F, Arancibia S, Tapia-arancibia L. Effect of aging on the expression of BDNF and TrkB isoforms in rat pituitary. Neurobiol Aging; 2007.28:1088-1098.

10.1016/j.neurobiolaging.2006.05.013

26. Sleiman SF, Henry J, Al-Haddad R, et al. Exercise promotes the expression of brain derived neurotrophic factor (BDNF) through the action of the ketone body $\beta$ hydroxybutyrate. $\quad$ Elife; 2016.5(6):1-21. DOI:10.7554/eLife. 15092

27. Nigam SM, Xu S, Kritikou JS, et al. Exercise and BDNF reduce $A \beta$ production by enhancing $\alpha$ - secretase processing of APP.J Neurochem; 2018.142(2):286-296. DOI:10.1111/jnc.14034.Exercise

28. Puche JE, Castilla-Cortázar I. Human conditions of insulin-like growth factor-I (IGF-I) deficiency. J Transl Med; 2012.10(1):1-29. DOI:10.1186/1479-5876-10-224

29. Le Grevès M, Le Grevès $P$, Nyberg F. Age-related effects of IGF-1 on the NMDA-, GH- and IGF-1receptor mRNA transcripts in the rat hippocampus. Brain Res Bull; 2005.65(5):369-374. DOI:10.1016/j.brainresbull.2005.01.012

30. Molina DP, Ariwodola OJ, Weiner JL, BrunsoBechtold JK, Adams MM. Growth hormone and insulin-like growth factor-I alter hippocampal excitatory synaptic transmission in young and old rats. Age (Omaha); 2013.35(5):1575-1587. DOI:10.1007/s11357-012-9460-4

31. Lee $\mathrm{CH}, \mathrm{Ahn} \mathrm{JH}$, Park JH, et al. Decreased insulin-like growth factor-I and its receptor expression in the hippocampus and somatosensory cortex of the aged mouse. Neurochem Res; 2014.39(4):770-776. DOI:10.1007/s11064-014-1269-3

32. Lee TK, Chen BH, Lee JC, et al. Age-dependent decreases in Insulin-like growth factor-I and its receptor expressions in the gerbil olfactory bulb. Mol Med Rep; 2018.17(6):8161-8166. DOI:10.3892/mmr.28.886

33. Ashpole NM, Sanders JE, Hodges EL, Yan H, Sonntag WE. Growth hormone, insulin-like growth factor-1 and the aging brain.Exp Gerontol;2016.68:76-81. DOI:10.1016/j.exger.2014.10.002.GROWTH

34. Trejo JL, Carro E, Lopez-Lopez C, Torres-Aleman I.
Role of serum insulin-like growth factor $\mathrm{I}$ in mammalian brain aging. Growth Horm IGF Res;2004.14:39-43. DOI:10.1016/j.ghir.2004.03.010

35. Shi L, Linville MC, Tucker EW, Sonntag WE, BrunsoBechtold JK. Differential effects of aging and insulinlike growth factor-1 on synapses in CA1 of rat hippocampus. Cereb Cortex;2005.15(5):571-577. DOI:10.1093/cercor/bhh158

36. Frystyk JAN. Exercise and the growth hormone insulin-like growth factor axis.Med Sci Sport Exerc; 2009.(30):58-66.

DOI:10.1249/MSS.0b013e3181b07d2d।

37. Carro E, Nuñez A, Busiguina S, Torres-Aleman I. Circulating insulin-like growth factor I mediates effects of exercise on the brain. J Neurosci;2000.20(8):29262933. DOI:10.1523/jneurosci.20-08-02926.2000

38. Trejo L, Carro E, Torres-Aleman I. Circulating insulinlike growth factor i mediates exercise-induced increases in the number of new neurons in the adult hippocampus. J Neurosci; 2001.21(5):1628-1634. DOI: 10.1523/JNEUROSCI.21-05-01628.2001

39. Glasper ER, Llorens-Martin M V., Leuner B, Gould E, Trejo JL. Blockade of insulin-like growth factor-I has complex effects on structural plasticity in the hippocampus. Hippocampus;2010.20(6):706-712. DOI:10.1002/hipo.20672

40. Peng Gao, PhD M, Fanxia Shen M, Rodney Allanigue Gabriel, BS, David Law M, et al. Attenuation of brain response to VEGF-mediated angiogenesis and neurogenesis in aged mice. Natl Institutes Heal; 2011.46(4):564-574.

DOI:10.1016/j.cortex.2009.08.003.Predictive

41. LeBlanc AJ, Shipley RD, Kang LS, Muller-Delp JM. Age impairs Flk-1 signaling and NO-mediated vasodilation in coronary arterioles. Am J Physiol - Hear Circ Physiol; 2008.295(6):2280-2289. DOI:10.1152/ajpheart.00541.2008

42. Fabel K, Fabel K, Tam B, et al. VEGF is necessary for exercise-induced adult hippocampal neurogenesis. Eur J Neurosci; 2003.18:2803-2812. DOI:10.1046/j.14609568.2003.03041.x

43. Rich B, Scadeng M, Yamaguchi M, Wagner PD, Breen EC. Skeletal myofiber vascular endothelial growth factor is required for the exercise training-induced increase in dentate gyrus neuronal precursor cells. J Physiol; 2017.595(17):5931-5943. DOI:10.1113/JP273994

44. Zhao Y, Pang Q, Liu M, Pan J, Xiang B. Treadmill Exercise promotes neurogenesis in ischemic rat brains via caveolin-1 / VEGF signaling pathways. Neurochem Res; 2017.42(2):387-397. DOI:10.1007/s11064-0162081-z

45. Han W, Song X, He R, et al. Epilepsy \&behavior VEGF regulates hippocampal neurogenesis and reverses cognitive de fi cits in immature rats after status epilepticus through the VEGF R2 signaling pathway. Epilepsy Behav; 2017.68:159-167. DOI:10.1016/j.yebeh.2016.12.007 